hep-ph/0107223

\title{
Spatially Anisotropic Four-Dimensional Gauge Interactions, Planar Fermions and Magnetic Catalysis
}

\author{
J. Alexandre, K. Farakos, G. Koutsoumbas \\ National Technical University of Athens, Zografou Campus, Athens GR157 \\ 80, Greece \\ and \\ N. E. Mavromatos \\ Department of Physics, Theoretical Physics, King's College London \\ Strand, London WC2R 2LS, U.K.
}

\begin{abstract}
We consider magnetic catalysis in a field-theoretic system of $(3+1)$-dimensional Dirac fermions with anisotropic kinetic term. By placing the system in a strong external magnetic field, we examine magnetically-induced fermion mass generation. When the coupling anisotropy is strong, in which case the fermions effectively localize on the plane, we find a significant enhancement of the induced mass gap compared to the isotropic four-dimensional case of quantum electrodynamics. As expected on purely dimensional grounds, the mass and critical temperature scale with the square root of the magnetic field. This phenomenon might be related to recent experimental findings on magnetically-induced gaps at the nodes of $d$-wave superconducting gaps in high-temperature cuprates.
\end{abstract}




\section{Introduction}

The phenomenon of magnetic catalysis, first suggested in [1], and subsequently developed also in [2], namely the dynamical generation of a fermion mass gap in the presence of external magnetic fields, has wide applications, ranging from particle physics and physics of the Early Universe [3] to condensed matter [4, 5, 6].

In the case of four-dimensional Abelian $U(1)$ gauge-Dirac-fermion models the magnetic catalysis phenomenon, for an external magnetic field $B$, is known [7] to yield a relatively small mass gap $m_{4 d}=m_{4 d}(B)$ (for standard low-energy electromagnetism, with fine structure constant of order 1/137) and an associated critical temperature of the same order $T_{s} \simeq m_{4 d}$. Such analyses pertain to Abelian gauge interactions with spatially isotropic couplings. It is the purpose of the present article to consider spatially anisotropic gauge interaction, in such a way that the gauge coupling on a spatial plane, defined by, say, the $x, y$ directions of a three-dimensional space, is much stronger than the coupling along the $z$-direction.

Physical motivation for such studies is provided by recent experimental findings in the physics of high-temperature layered superconductors [8]. According to such experiments, one looks at the thermal conductivity properties of a sample of high-temperature superconductors in the superconducting phase, in the presence of strong external magnetic fields (with intensities up to a few Tesla). The high-temperature superconducting samples are known to be strongly type II, and so the magnetic field lines penetrate the material significantly. Moreover, such materials are known to be $d$-wave superconductors with a layered structure of $\mathrm{Cu}-\mathrm{O}$ planes, characterized by nodes in their superconducting excitation gaps. The experiments of [8] have demonstrated that below a given temperature, which is smaller than the critical temperature of the superconductor, and which scales as the square root of the applied magnetic field, there are plateaux in the thermal conductivity diagrams. The plateaux indicate the opening of gaps at the nodes below this new 'critical' temperature (not to be confused with the superconducting/normal phase critical temperature) .

In ref. [4 we suggested that this phenomenon might be a straightforward application of the magnetic catalysis phenomenon [1, 2, 9] to the threedimensional effective problem of physics near the nodes. Indeed, lineariz-

ing the excitations about such nodes one obtains, upon the assumption of 
spin-charge separation [10], a relativistic electrically-charged fermion (Dirac) system, coupled to statistical gauge fields in the presence of external electromagnetic fields. The strong external magnetic field induces the opening of a nodal holon gap, which scales with the magnetic field $B$. The experiments of [8] have shown a square root scaling with $B$. In the models of [曰, 11], the statistical gauge fields represent effective spin-spin antiferromagnetic interactions, which are believed by many to be relevant for the physics of the high-temperature superconductivity. In ref. [4], and in all the subsequent works [5, 6], where various interactions, including four-fermi, among the holons have been considered, the model systems have been assumed to live exclusively in $(2+1)$-dimensions, ignoring completely any four-dimensional physics effects.

This may not be physically correct, especially from the point of view of the gauge and electromagnetic interactions, which are known to be fully four-dimensional 円. Moreover, the superconducting gaps are actually fourdimensional, although strongly anisotropic, i.e. with dominant components along the $\mathrm{Cu}-\mathrm{O}$ planes, but with suppressed, however non-vanishing, components along the perpendicular (interplanar) direction.

It is the purpose of this article therefore to consider such an anisotropic four-dimensional situation and study the consequences for magnetically induced dynamical mass generation for the fermionic excitations. From the condensed-matter point of view, such excitations may be the (continuum limit of) holons, carrying the electric charge only, but no actual spin (in the microscopic sense). In ref. [4] the holons were assumed purely threedimensional (planar), as a result of the localization of their wavefunctions on the superconducting planes. This is a basic feature assumed to characterize the microscopic physics behind spin-charge separation [10]. In the present article the fermions will be assumed four-dimensional, but with anisotropic kinetic terms, which allows for interplanar hoping. In the strong anisotropic case one should recove the three-dimensional case. In this sense one should have the extension of the concept of spin-charge separation to four dimensions, in the strongly anisotropic case. This, of course, does not apply to the concept of fractional statistics [11], which is only an exclusive feature of

\footnotetext{
${ }^{1}$ For instance, interlayer couplings via magnetic spin-spin interactions are known to exist in the planar high-temperature superconducting materials, and, of course, the electromagnetic interactions are fully four dimensional.
} 
the three-dimensional case. However, there may be a different scenario, in which the fermions discussed here are viewed as related to real nodal electron excitations (carrying both spin and charge), capable of interlayer hopping. It is because of this latter interpretation that, when we discuss in section 5 the non-relativistic situation of relevance to condensed matter, we shall ignore any statistical gauge interactions among the fermions, keeping only the electromagnetic interactions.

In case one assumes the existence of four-dimensional holons, by extending the concept of spin-charge separation to four-dimensions, allowing, though, for weak interlayer hopping of holons, one might encounter a situation relevant to the fully relativistic case discussed in the next section, in which the gauge fluctuations represent statistical gauge fields. In such a case one may even encounter non-abelian gauge interactions. Indeed, in the physical models of [11], the statistical gauge fields are non-Abelian, of $S U(2)$ type, due to an underlying 'particle-hole symmetric' formulation of the spin-charge separation ansätze. Such interactions are not responsible for the opening of holon mass gaps, because of the fermion spectrum in the threedimensional models of [11]. It is the statistics-changing $U_{S}(1)$-interaction, exclusive for three-dimensional systems, which is strong enough to generate such gaps. Unfortunately the $U_{S}(1)$ interaction does not have an analogue in four-dimensions. Thus, although probably relevant for the superconductivity scenaria, nevertheless such statistical interactions may be not directly relevant for the four-dimensional physics underlying the findings of [ 8 ].

For our purposes below, therefore, we shall ignore such non-Abelian statistical interactions, and concentrate rather exclusively on the rôle of real electromagnetic interactions, described by a potential $A_{\mu}$, in inducing, under the influence of a strong external magnetic field, a mass gap for the nodal holons. We shall assume, however, that the electromagnetic interactions are screened along the $z$-direction (interplanar), and in this sense we shall treat the gauge coupling $e$ as spatially anisotropic. A subtlety of this model is the relativistic non-invariance of the electromagnetic interactions in the presence of holons. The fermion (holon or, even, charged electron excitations) part is a relativistic system describing nodal excitations, but for such a system the rôle of the limiting speed of 'light' is played by the fermi velocity $v_{F}$ of the nodes. On the other hand, the real electromagnetic field propagates with the velocity of light $c \gg v_{F}$ (for realistic systems $c \sim 10^{4} v_{F}$, but for us $v_{F}$ will be considered as a phenomenological parameter). When the combined system 
is expressed in terms of the fermi velocity, the electromagnetic interaction will be non relativistic, with the velocity of light $c$ appearing explicitly in the Maxwell action. This leads to a non-relativistic form for the photon propagator in the environment of holons or equivalently to a non-relativistic form for the fermion propagator if we rescale the fields.

Our approach in the present article will be field theoretic, and we shall not attempt to make further contact with the condensed-matter systems, apart from the very generic features mentioned above. However, as we shall see, our findings are interesting enough, and indeed may be of use in attempts to explain the phenomenon of [8] by means of the magnetic catalysis mechanism of relativistic fermions [4, 5, 6]. A brief discussion on such 'phenomenology' will appear at the end of our article.

At this stage the reader's attention is drawn to a very interesting recent work [12], which analyses the phenomenon of chiral symmetry breaking on a brane domain wall, embedded in a higher-dimensional space time, in the absence of any external fields. This situation is different from the one discussed here, not only because there is no external field, but also because in the work of [12] the fermions are completely localized on the brane, while the gauge fields propagate in the bulk. In contrast, in our case we allow interlayer hopping for fermions, which is anisotropic, and also we have anisotropic gauge couplings. In the scenaria of [12], the fermions could represent purely threedimensional holons of planar high-temperature cuprates, localised on the $\mathrm{Cu}-\mathrm{O}$ planes. In such scenaria, therefore, the spin-charge separation would be an exclusive feature of the planes, not extended (even in the strongly anisotropic sense) to four dimensions, but the statistical interactions (represented by the gauge fields) could extend to interplanar coupling situations. This would make an important physical difference from the scenaria discussed in the present article.

The structure of the article is as follows: in section 2 we study the magnetic catalysis phenomenon in relativistic gauge-fermions systems in the presence of anisotropic four-dimensional gauge couplings. In section 3 we demonstrate that, in the strongly anisotropic case, there is a significant enhancement of the induced mass gap $m_{d y n}$ (on the plane), as compared with the isotropic case. In section 4 we study the system at finite temperature, and compute the critical temperature $T_{c}$ above which the magnetically-induced mass gap disappears. We demonstrate that $T_{c} \simeq m_{d y n}$, as expected on natural grounds. The square-root scaling with the magnetic field intensity is also 
demonstrated. In section 5 we attempt to make contact with realistic condensed matter systems, and the experiments of [8], by repeating the above analysis but for a non-relativistic fermion system, coupled to a relativistic electromagnetic field, again with anisotropic couplings, whose quantum fluctuations are taken into account in the absence of any other interactions. The rôle of the limiting speed of light for the fermions (holons or electricallycharged nodal excitations) is played by the fermi velocity $v_{F}$, while, the speed of light is $c>v_{F}$. In this case the ratio $v_{F} / c<1$ is a phenomenological parameter of the model. The main features of the previous fully-relativistic case are maintained, especially as far as the enhancement of the fermion gap is concerned in the strongly anisotropic case. However, there are extra suppression factors by powers of $v_{F} / c$, relative to the relativistic case of section 2. Conclusions and some discussion, with relevance to 'phenomenology' of high-temperature superconductivity, are presented in section 6. Some formal aspects of the Schwinger-Dyson analysis for the anisotropic case are presented in an Appendix.

\section{Anisotropic gap equation}

The Lagrangian density which includes the anisotropy is, in the absence of an external field

$$
\mathcal{L}=-\frac{1}{4} F_{\mu \nu} F^{\mu \nu}+\bar{\psi}\left[i \not \partial-e A-x\left(i \partial_{3}-e A_{3}\right) \gamma^{3}-m\right] \psi
$$

where $\not \partial=\gamma^{\mu} \partial_{\mu}, \mu, \nu=0,1, \ldots, 3, \gamma^{\mu}$ are four-dimensional $4 \times 4$ Dirac matrices, $\psi$ are four-component spinors, and the parameter $x$ controls the anisotropy. The case $x=0$ corresponds to a totally isotropic situation (the usual quantum electrodynamics (QED)), while $x=1$ corresponds to a totally anisotropic one: the gauge field lives in $3+1$ dimensions whereas the fermions are effectively localized in $2+1$ dimensions. We choose the $\gamma$ matrices $\gamma^{i}, i=0,1,2$ diagonal and the matrix $\gamma^{3}$ non-diagonal, as in [5]. In the totally anisotropic case $x=1, \gamma^{3}$ does not appear in the action and the other $\gamma$ matrices decompose in two sets of two-component Dirac matrices, appropriate for the irreducible Dirac algebra in $(2+1)$-dimensions. In such a case the four-component spinors also decompose to an even number ( 2 for a single flavour) of two-component $(2+1)$-dimensional spinors, and one recovers the 
planar case, after integration over the third dimension 9 .

It should be stressed that Eq.(1) of course respects the gauge invariance. With such a Lagrangian, and in the absence of external field, the bare fermion propagator is given by

$$
i S^{-1}(p)=\not p-x p_{3} \gamma^{3}-m .
$$

As an illustration of the effect of the anisotropy, we can compute the propagation of a fermion in the direction 3. Let us define in the Euclidean space

$$
\begin{aligned}
\phi\left(r_{3}\right) & =\operatorname{tr} \int d r_{4} d r_{2} d r_{1}<0|\bar{\psi}(0) \psi(r)| 0> \\
& =\operatorname{tr} \int d r_{4} d r_{2} d r_{1} \int \frac{d^{4} p}{(2 \pi)^{4}} S(p) e^{i p r} \\
& =\operatorname{tr} \int \frac{d p_{3}}{2 \pi} S\left(0,0, p_{3}, 0\right) e^{i p_{3} r_{3}},
\end{aligned}
$$

i.e. the propagation rate of the fermions from the $2+1$ dimensional worlds $r_{3}=0$ and $r_{3} \neq 0$. A straightforward computation leads to

$$
\phi\left(r_{3}\right)=\frac{2}{1-x} \exp \left(-\frac{m\left|r_{3}\right|}{1-x}\right),
$$

which shows that as the anisotropy increases, the fermion propagation in the direction 3 decreases exponentially. Eventually, as $x \rightarrow 1$, this propagation vanishes if $r_{3} \neq 0$. This corresponds to an effective delta-function in $r_{3}$ which kills the $r_{3}$ integration in the action leading to a three-dimensional theory.

We derive in the appendix the Schwinger-Dyson equation for the fermion propagator, taking into account the anisotropy parameter $x$ and find (we do not write the space-time indices)

$$
G=S-4 \pi \alpha \int S \gamma^{\mu} G \Lambda^{\nu} G D_{\mu \nu}+x 4 \pi \alpha \int S \gamma^{3} G \Lambda^{\nu} G D_{3 \nu}
$$

where $G$ is the full fermion propagator, $D_{\mu \nu}$ the full photon propagator and $\Lambda^{\mu}$ the full vertex which satisfies at the tree level

\footnotetext{
${ }^{2}$ In the physical case of relevance to condensed matter, the third dimension may extend between two $\mathrm{Cu}-\mathrm{O}$ layers, which play the rôle of boundaries of the available space. In such a case the extra (third) dimension is integrated in the bulk space between these boundaries. In the analysis below we shall not assume explicitly such geometries, but rather treat the anisotropic four-dimensional case in a generic sense, specified by the Lagrangian (1).
} 


$$
\begin{aligned}
& \Lambda_{\text {tree }}^{\mu}=\gamma^{\mu} \quad \text { if } \mu \neq 3 \\
& \Lambda_{\text {tree }}^{3}=(1-x) \gamma^{3}
\end{aligned}
$$

We use the usual definition of the fine structure constant: $e^{2}=4 \pi \alpha$.

In the presence of an external, constant and homogenous magnetic field in the direction 3 , we can choose an external gauge such that $A_{3}^{\text {ext }}=0$ for which the parameter $x$ will not couple to the external field and thus will only play a role in the substitution $p_{3} \rightarrow(1-x) p_{3}$. The lowest Landau level (LLL) approximation [9] for the fermion propagator will be then

$$
S^{L}(y, z)=e^{i e y^{\mu} A_{\mu}^{e x t}(z)} \tilde{S}^{L}(y-z),
$$

where the Fourier transform of the translational invariant propagator $\tilde{S}^{L}$ is

$$
\tilde{S}^{L}(p)=e^{-p_{\perp}^{2} /|e B|} \frac{i\left(1-i \gamma^{1} \gamma^{2}\right)}{p_{0} \gamma^{0}+(1-x) p_{3} \gamma^{3}-m}
$$

where $p_{\perp}^{2}=p_{1}^{2}+p_{2}^{2}$ is the transverse momentum squared. We will take $m=0$ for the bare propagator (not taking into account the interaction with the dynamical gauge field) and $m=m\left(p_{0}, p_{3}\right)$, the dynamical self energy which depends only on the longitudinal momenta in the LLL approximation, for the full propagator. The integral equation describing the magnetic catalysis in the LLL approximation contains only the components $D_{00}$ and $D_{33}$ of the photon propagator [9], due to the spin projector $\left(1-i \gamma^{1} \gamma^{2}\right) / 2$ in the fermion propagator (8). These two components lead to equal contributions and thus the second integral in the Schwinger-Dyson equation (5) which is proportional to $x$ gives half the contribution of the first integral. The integral equation reads then, if we neglect the corrections to the vertex,

$$
G^{L}=S^{L}-4 \pi \alpha\left(1-x+\frac{x^{2}}{2}\right) \int S^{L} \gamma^{\|} G^{L} \gamma^{\|} G^{L} D_{\|,\|},
$$

where $\|$ denotes the longitudinal components. The final integral equation is found by making the substitution $p_{3} \rightarrow(1-x) p_{3}$ in the fermion propagators appearing in the integral of Eq.(9) which has already been computed in the isotropic case [1]. If we rescale all the quantities homogenous to a mass by $\sqrt{|e B|}$, we have in the Euclidean space 


$$
\begin{aligned}
\mu\left(k_{3}, k_{4}\right)= & \left(1-x+\frac{x^{2}}{2}\right) \frac{\alpha}{\pi^{2}} \int \frac{d p_{3} d p_{4} \mu\left(p_{3}, p_{4}\right)}{(1-x)^{2} p_{3}^{2}+p_{4}^{2}+\mu^{2}\left(p_{3}, p_{4}\right)} \\
& \times \int_{0}^{\infty} \frac{d u e^{-u}}{2 u+\left(p_{3}-k_{3}\right)^{2}+\left(p_{4}-k_{4}\right)^{2}},
\end{aligned}
$$

where $\mu$ is the dimensionless self-energy and $u$ a dimensionless transverse momentum squared. The momenta $p_{3}$ and $p_{4}$ play a symmetric role in the photon propagator whereas they enter non-symmetrically in the fermion propagator, which leads to a quite difficult analysis of the integral equation

if we wish to take into account the momentum dependence of the fermion self energy. We know that this momentum dependence is essential in $3+1$ dimensions [9] but not in $2+1$ dimensions, as long as we consider a qualitative description [13]. In this paper we wish to give a qualitative description of the magnetic catalysis in the strongly anisotropic regime $1-x<<1$ and thus will make the constant self-energy approximation in which the integral equation finally reads

$$
1=\left(1-x+\frac{x^{2}}{2}\right) \frac{\alpha}{\pi^{2}} \int \frac{d p_{3} d p_{4}}{(1-x)^{2} p_{3}^{2}+p_{4}^{2}+\mu_{0}^{2}} \int_{0}^{\infty} \frac{d u e^{-u}}{2 u+p_{3}^{2}+p_{4}^{2}},
$$

or, when we make the angular integration,

$$
1=\left(1-x+\frac{x^{2}}{2}\right) \frac{\alpha}{\pi} \int_{0}^{\infty} \frac{d \rho}{\sqrt{\left(\rho+\mu_{0}^{2}\right)\left[(1-x)^{2} \rho+\mu_{0}^{2}\right]}} \int_{0}^{\infty} \frac{d u e^{-u}}{2 u+\rho}
$$

The analysis of the approximation (11) in the isotropic regime $x<<1$ will not lead to a reliable quantitative dynamical mass $\mu_{0}$ but will show us the qualitative tendency of $\mu_{0}$ to increase when the anisotropy increases.

\section{Enhancement of the dynamical mass}

In this section we will show that the anisotropy generates a considerably enhanced mass gap, compared to the isotropic situation where we know that in the constant self-energy approximation [囬], [2], 


$$
\mu_{0}(x=0) \simeq \sqrt{2} \exp \left(-\sqrt{\frac{\pi}{\alpha}}\right) .
$$

To see the increase of the dynamical mass when $x$ increases, we make an expansion of the integral equation (11) up to the order $x^{2}$ and find

$$
1=\frac{\alpha}{\pi^{2}} \int_{0}^{\infty} d u e^{-u}\left\{\mathcal{I}_{1}+x\left(2 \mathcal{I}_{2}-\mathcal{I}_{1}\right)+x^{2}\left(4 \mathcal{I}_{3}-3 \mathcal{I}_{2}+\frac{1}{2} \mathcal{I}_{1}\right)+\ldots\right\},
$$

where

$$
\begin{aligned}
\mathcal{I}_{1} & =\int \frac{d p_{3} d p_{4}}{\left(p_{3}^{2}+p_{4}^{2}+\mu_{0}^{2}\right)\left(p_{3}^{2}+p_{4}^{2}+2 u\right)} \\
& =\pi \frac{\ln \left(2 u / \mu_{0}^{2}\right)}{2 u-\mu_{0}^{2}} \\
\mathcal{I}_{2} & =\int \frac{d p_{3} d p_{4} p_{3}^{2}}{\left(p_{3}^{2}+p_{4}^{2}+\mu_{0}^{2}\right)^{2}\left(p_{3}^{2}+p_{4}^{2}+2 u\right)} \\
& =\frac{\pi}{2} \frac{2 u \ln \left(2 u / \mu_{0}^{2}\right)-2 u+\mu_{0}^{2}}{\left(2 u-\mu_{0}^{2}\right)^{2}} \\
\mathcal{I}_{3} & =\int \frac{d p_{3} d p_{4} p_{3}^{4}}{\left(p_{3}^{2}+p_{4}^{2}+\mu_{0}^{2}\right)^{3}\left(p_{3}^{2}+p_{4}^{2}+2 u\right)} \\
& =\frac{3 \pi}{16} \frac{8 u^{2} \ln \left(2 u / \mu_{0}^{2}\right)+\left(\mu_{0}^{2}-6 u\right)\left(2 u-\mu_{0}^{2}\right)}{\left(2 u-\mu_{0}^{2}\right)^{3}} .
\end{aligned}
$$

Despite their appearance, the integrals (15) are converging when $2 u \rightarrow \mu_{0}^{2}$, what can be checked by expanding the logarithms around $2 u=\mu_{0}^{2}$.

For the integration over the transverse momentum $u$, we write that for $\mu_{0}<<1$ :

$$
\begin{aligned}
& \int_{0}^{\infty} d u \mathcal{I}_{1} e^{-u}=\pi \int_{0}^{\infty} d u e^{-u} \frac{\ln \left(2 u / \mu_{0}^{2}\right)}{2 u-\mu_{0}^{2}} \\
& \simeq \frac{\pi}{2} \int_{0}^{2 / \mu_{0}^{2}} d u \frac{\ln u}{u-1} \simeq \frac{\pi}{2} \int_{1}^{2 / \mu_{0}^{2}} d u \frac{\ln u}{u} \\
& =\pi \ln ^{2}\left(\frac{\sqrt{2}}{\mu_{0}}\right)
\end{aligned}
$$


Similar approximations lead to

$$
\begin{aligned}
\int_{0}^{\infty} d u \mathcal{I}_{2} e^{-u} & \simeq \frac{\pi}{2} \ln ^{2}\left(\frac{\sqrt{2}}{\mu_{0}}\right) \\
\int_{0}^{\infty} d u \mathcal{I}_{3} e^{-u} & \simeq \frac{3 \pi}{8} \ln ^{2}\left(\frac{\sqrt{2}}{\mu_{0}}\right),
\end{aligned}
$$

and give the following equation for the dynamical mass

$$
\left(1+\frac{x^{2}}{2}+\ldots\right) \ln ^{2}\left(\frac{\sqrt{2}}{\mu_{0}}\right)=\frac{\pi}{\alpha} .
$$

We have then for the dimensionful dynamical mass, to order $x^{2}$,

$$
m_{d y n}(x) \simeq \sqrt{2} \sqrt{|e B|} \exp \left\{-\sqrt{\frac{\pi}{\alpha}}\left(1-\frac{x^{2}}{4}\right)\right\} .
$$

We see then that the anisotropy $(x>0)$ has the effect to increase the dynamical mass.

Let us now come to the totally anisotropic regime and compute the dynamical mass obtained for $x=1$. In this case, we find from (12)

$$
1=\frac{\alpha}{2 \pi} \int_{0}^{\infty} \frac{d \rho}{\sqrt{\rho+1}} \int_{0}^{\infty} \frac{d u e^{-u}}{2 u+\rho \mu_{0}^{2}} .
$$

To perform the integration over $\rho$, we suppose that $2 u>\mu_{0}^{2}$, which consists in neglecting the interval $\left[0, \mu_{0}^{2} / 2\right]$ in the integration over $u$, which will be justified since we will find $\mu_{0}^{2}<<1$. We have then

$$
1 \simeq \frac{\alpha}{2 \mu_{0}} \int_{\mu_{0}^{2} / 2}^{\infty} d u e^{-u} \frac{1}{\sqrt{2 u-\mu_{0}^{2}}} \simeq \frac{\alpha}{4} \int_{1}^{2 / \mu_{0}^{2}} d u \frac{1}{\sqrt{u-1}}
$$

such that we finally obtain for the dimensionful dynamical mass

$$
m_{d y n}(x=1) \simeq \frac{\alpha}{\sqrt{2}} \sqrt{|e B|} .
$$

This result shows that the anisotropy plays a fundamental role in the generation of a big mass gap since $m_{d y n}(x=1)>>m_{d y n}(x=0)$. We show in figure 1 the dynamical mass as a function of $x$, for a given value of the 


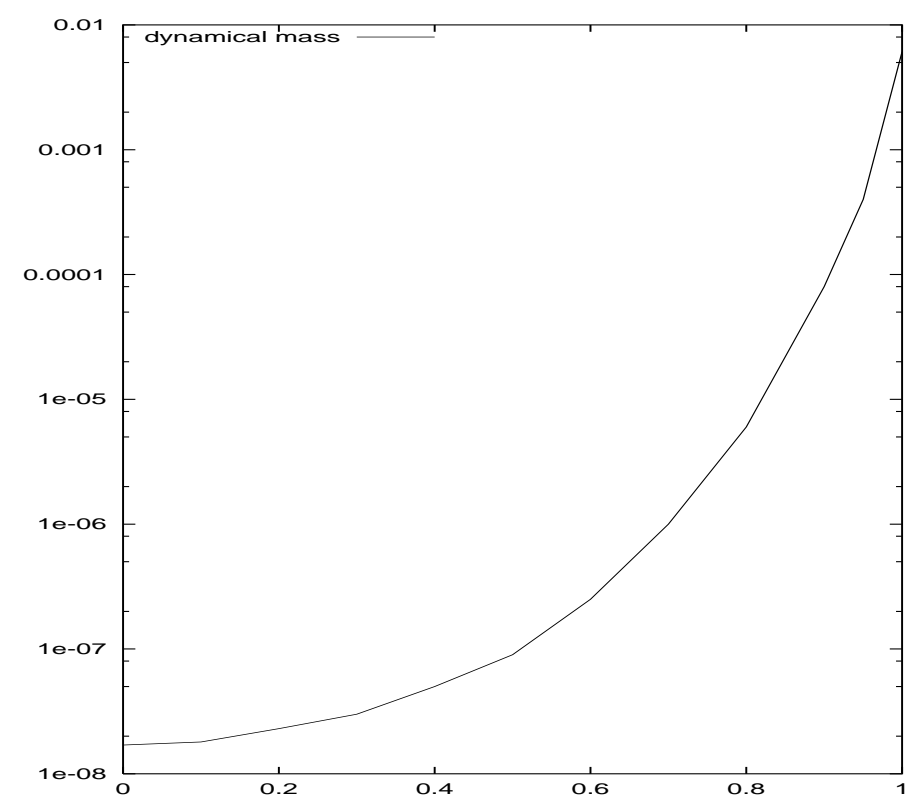

Figure 1: $\mu_{0}$ versus $x$ for $\alpha=.01$

coupling $\alpha$. This figure has been done with the numerical study of Eq.(12) and we note the logarithmic scale, showing the exponential increase of the dynamical mass. The analytical results (19) and (22) are confirmed by the curve in the asymptotic limits $x<<1$ and $1-x<<1$.

\section{Critical temperature in the anisotropic regime}

We now turn to the finite temperature treatment of the problem so as to find the critical temperature when $x=1$.

The integral equation at finite temperature is obtained by the usual substitutions $p_{4} \rightarrow \omega_{l}=(2 l+1) \pi t$ and $\int d p_{4} \rightarrow 2 \pi t \sum_{l}$ in the equation (11), and $t=T / \sqrt{|e B|}$ is the dimensionless temperature. We just have to pay attention to the photonic momentum $p_{4}$ which has to be changed by $\omega_{l}-\omega_{0}$, that is to say to a bosonic Matsubara mode. We obtain then the following equation for the critical temperature defined by $\mu_{0}\left(t_{c}\right)=0$ : 


$$
1=\frac{\alpha}{\pi} \int d p_{3} \int_{0}^{\infty} d u e^{-u} t_{c} \sum_{l=-\infty}^{l=\infty} \frac{1}{\omega_{l}^{2}\left[2 u+p_{3}^{2}+\left(\omega_{l}-\omega_{0}\right)^{2}\right]}
$$

where $\omega_{0}=\pi t_{c}$. The summation over the dimensionless Matsubara modes $\omega_{l}$ is done with the usual contour deformation trick detailed for example in [13] and gives

$$
\begin{aligned}
& t_{c} \sum_{l=-\infty}^{l=\infty} \frac{1}{\omega_{l}^{2}\left[2 u+p_{3}^{2}+\left(\omega_{l}-\omega_{0}\right)^{2}\right]}=\frac{1}{4 t_{c}} \frac{1}{2 u+p_{3}^{2}+\omega_{0}^{2}} \\
& +\frac{1}{2 \sqrt{2 u+p_{3}^{2}}} \operatorname{coth}\left(\frac{\sqrt{2 u+p_{3}^{2}}}{2 t_{c}}\right) \frac{\omega_{0}^{2}-2 u-p_{3}^{2}}{\left(\omega_{0}^{2}+2 u+p_{3}^{2}\right)^{2}} .
\end{aligned}
$$

We suppose that the coth is close to 1 since $t_{c}<<1$. This is actually not valid when $2 u+p_{3}^{2}<<1$ but this region is negligible in the remaining integrals. The integral equation reads then

$$
\begin{aligned}
1= & \frac{\alpha \pi}{8} \int_{0}^{\infty} d u e^{-u \omega_{0}^{2} / 2} \frac{1}{\sqrt{1+u}} \\
& +\frac{\alpha}{\pi} \int_{0}^{\infty} d u e^{-u \omega_{0}^{2} / 2} \int_{0}^{\infty} d v \frac{1-u-v^{2}}{\left(1+u+v^{2}\right)^{2} \sqrt{u+v^{2}}}
\end{aligned}
$$

The dominant contribution in Eq.(25) comes from the first term when $t_{c}<<$ 1 since in the second term, the integration over $v$ gives

$$
\begin{aligned}
& \int_{0}^{\infty} d v \frac{1-u-v^{2}}{\left(1+u+v^{2}\right)^{2} \sqrt{u+v^{2}}} \\
= & -\frac{1}{1+u}+\frac{1}{4(1+u)^{3 / 2}} \ln \left(\frac{2+u+2 \sqrt{1+u}}{2+u-2 \sqrt{1+u}}\right),
\end{aligned}
$$

which leads to higher powers of $t_{c}$. The critical temperature is then given by

$$
1 \simeq \frac{\alpha \pi}{8} \int_{0}^{2 / \omega_{0}^{2}} d u \frac{1}{\sqrt{1+u}}
$$

such that the dimensionful critical temperature is finally 


$$
T_{c}(x=1) \simeq \frac{\alpha}{2 \sqrt{2}} \sqrt{|e B|}=\frac{1}{2} m_{d y n}(x=1) .
$$

This result confirms what was expected, i.e. the critical temperature is of the order of the dynamical mass at zero temperature. The same conclusion was found in [7] for isotropic QED.

\section{Non-Relativistic Gauge Field-Fermion Models and an Application to Condensed Matter}

So far we have examined a relativistic system, in which the gauge interactions may be assumed distinct from the real electromagnetic interactions. From a condensed matter view point, such systems may have some relevance to effective gauge theories of $t-j$ models, with intrasublattice hopping, pertaining to spin-charge separation scenaria applied, however, to nodal $d$-wave excitations [14].

In this section we shall consider a problem which might be considered as more "realistic", in the sense of being connected directly to the observed physics of high-temperature superconductors, in view of the recent experimental findings of [8]. We shall not assume ad hoc any statistical interaction, but we shall continue to apply a spin-charge separation scenario [10 for the nodal excitations of the high-temperature $d$-wave superconductors.

In this case, the electrically-charged excitations (holons) around the nodes of a $d$-wave supercondcuting gap will be represented by $(3+1)$-dimensional Dirac fermions coupled to an electromagnetic field, described by a potential $A_{\mu}=\left(A_{0}, A_{i}\right)$. The four-dimensional nature of the fermions implies the possibility of interplanar hopping for such excitations in the materials, which is a realistic feature. We shall consider the case where the electromagnetic field has a background, corresponding to a constant magnetic field along the $z$ direction, and four-dimensional quantum fluctuations around it, and assume no further interactions among the holons. However, the electromagnetic coupling $e$ will be assumed screened along the $z$-direction, i.e. the coupling is anisotropic. Such a screening may be provided by a combination of both the chemistry and geometry of the planar materials, which consist of layers 
of $\mathrm{Cu}-\mathrm{O}$, with lattice spacing of 5 Angströms, while the interlayer distance is much larger, of the order of 100 Angströms. In the interlayer space there are doping atoms etc., which may be responsible for an effective charge screening.

From a formal view point we might think of the effective electric-charge anisotropy as follows: the coupling constant $e^{2} / 4 \pi$ entering the problem, as a result of quantum fluctuations of the electromagnetic field, is actually a running coupling constant, which depends on the available energy. If the latter is viewed as the inverse of a characteristic distance for the problem, we then observe that, as a result of the relatively large separation between two holons (which are the relevant degrees of freedom for the problem) at different layers, assumed perpendicular to the $z$ direction, the resulting effective charge (along the $z$ direction) lies in the infrared regime, and as such is much weaker than the effective charge between holons in the same layer and actually at characteristic distances of the order of the inverse of the magnetically-induced mass gap, which we are interested in for the purposes of the present work. In high-temperature materials this distance, i.e. the magnetic coherence length of nodal charged excitations, is short enough and of the order of a few angströms, which justifies the assumed strong anisotropy of the electric charge.

With these in mind, we consider the following model. The photon kinetic term is the usual one

$$
\mathcal{L}_{G}=-\frac{1}{4} F_{\mu \nu} F^{\mu \nu},
$$

with $\partial_{0}=\partial / \partial t(c=1)$ and the free fermion kinetic term is

$$
\mathcal{L}_{F}=\bar{\psi}\left(i \gamma^{0} \tilde{\partial}_{0}+i \gamma^{k} \partial_{k}\right) \psi
$$

with $\tilde{\partial}_{0}=\partial / \partial\left(v_{F} t\right)$. Since the fermion density and current are (the notation $\vec{A}$ denotes spatial three vectors):

$$
\begin{aligned}
\rho & =\psi^{\dagger} \psi \\
\overrightarrow{\mathrm{J}} & =v_{F} \bar{\psi} \vec{\gamma} \psi,
\end{aligned}
$$

the interaction between the fermions and the gauge field is

$$
\mathcal{L}_{I}=e \bar{\psi} \gamma^{0} A_{0} \psi+e v_{F} \bar{\psi} \vec{\gamma} \cdot \vec{A} \psi
$$


such that the final Lagrangian will contain the following fermionic part

$$
\bar{\psi}\left[\eta\left(i \partial_{0} \gamma^{0}-g A_{0} \gamma^{0}\right)+\left(i \partial_{k} \gamma^{k}-g A_{k} \gamma^{k}\right)-x\left(i \partial_{3} \gamma^{3}-g A_{3} \gamma^{3}\right)\right] \psi
$$

where we define $\eta=1 / v_{F}, g=e v_{F}$ and $k=1,2,3$. The bare fermion propagator is then, in the absence of external field,

$$
i S^{-1}(p)=\eta p_{0} \gamma^{0}+p_{k} \gamma^{k}-x p_{3} \gamma^{3}-m
$$

It is straightforward to see from the derivation shown in the appendix that the Schwinger-Dyson equation changes to

$$
\begin{aligned}
G= & S-\eta 4 \pi \alpha \int S \gamma^{0} G \Lambda^{\nu} G D_{0 \nu} \\
& -4 \pi \alpha \int S \gamma^{k} G \Lambda^{\nu} G D_{k \nu}+x 4 \pi \alpha \int S \gamma^{3} G \Lambda^{\nu} D_{3 \nu}
\end{aligned}
$$

where

$$
\begin{aligned}
\Lambda_{\text {tree }}^{\nu} & =\gamma^{\nu} \quad \text { if } \nu \neq 0,3 \\
\Lambda_{\text {tree }}^{0} & =\eta \gamma^{0} \\
\Lambda_{\text {tree }}^{3} & =(1-x) \gamma^{3}
\end{aligned}
$$

and $g^{2}=4 \pi \alpha$. The integral equation corresponding to (11) is finally

$$
1=\left[\eta^{2}+(1-x)^{2}\right] \frac{\alpha}{2 \pi^{2}} \int \frac{d p_{3} d p_{4}}{(1-x)^{2} p_{3}^{2}+\eta^{2} p_{4}^{2}+\mu_{0}^{2}} \int_{0}^{\infty} \frac{d u e^{-u}}{2 u+p_{3}^{2}+p_{4}^{2}}
$$

This last equation reads for $x=1$

$$
1=\frac{\alpha}{2 \pi^{2}} \int \frac{d p_{3} d p_{4}}{p_{4}^{2}+\left(\mu_{0} / \eta\right)^{2}} \int_{0}^{\infty} \frac{d u e^{-u}}{2 u+p_{3}^{2}+p_{4}^{2}},
$$

which is the equation (20) with the substitution $\mu_{0} \rightarrow \mu_{0} / \eta$. Since $\eta>>1$, the condition $\mu_{0} / \eta<<1$ is still valid and the result is then for the dimensionful dynamical mass 


$$
m_{d y n} \simeq v_{F}^{3 / 2} \frac{e^{2}}{4 \pi \sqrt{2}} \sqrt{|e B|}
$$

For the finite temperature case, the equation (23) does not change (the factors $\eta$ cancel when $x=1$ ) and the critical temperature is then

$$
T_{c} \simeq v_{F}^{5 / 2} \frac{e^{2}}{8 \pi \sqrt{2}} \sqrt{|e B|}
$$

It is important to notice that both the the dynamical mass (39), and the associated critical temperature (40), are proportional to a suppression factor

$v_{F}^{3 / 2}=\mathcal{O}\left(10^{-6}\right)$ and $v_{F}^{5 / 2}=\mathcal{O}\left(10^{-10}\right)$ respectively, compared to the previoulsy considered relativistic case. This may have important phenomenological implications when one attempts to compare the scenaria advocated here with realistic condensed-matter situations [8]. We shall not do such analyses here, given that at present we lack a detailed derivation of the continuum models discussed above from microscopic condensed matter models. This is essential for providing the correct order of magnitude of the various coupling constants, such as gauge, hopping elements etc., entering the model.

\section{Discussion}

In this work we have discussed the case of four-dimensional anisotropic fermions coupled to anisotropic-coupling Abelian gauge fields, and external magnetic fields. By considering fluctuations of the gauge fields we have considered the magnetic catalysis phenomenon, i.e. the dynamical mass generation for fermions under the influence of strong external magnetic fields. We have considered two cases: the fully relativistic case, in which fermions and fluctuating gauge fields are relativistic, and the case where the photon fields are relativistic, but the fermion part of the Lagrangian is non relativistic, with a fermi velocity $v_{F} \neq c$ ( $c$ is the speed of light) playing the rôle of the limiting velocity for the fermionic part.

In both cases a strong planar anisotropy has been assumed. The analysis has shown a significant enhancement of the induced mass gap, relative to the isotropic four-dimensional case. In the non-relativistic case, however we find that the induced mass gap is found suppressed by some power of the fermi velocity $v_{F}<c$, as compared to the relativistic case. In realistic situations, 
$v_{F} / c \sim 10^{-4}$, and the suppression factors are significant. In both cases, the mass gap and the associated critical temperature, above which the magnetic catalysis disappears due to thermal disorder, exhibit a square-root scaling with the magnetic field intensity, as expected on natural grounds.

The non relativistic case, may be related to the situation encountered in high-temperature superconductors in the experiments of [8]. In such a case, the fermions are electrically charged excitations, which can represent excitations about the nodes of the $d$-wave superconducting gaps. The relativistic nature of the excitations is due to the nodal structure. These excitations may be holons, capable though of interlayer hopping, or could be real (nodal) electron degrees of freedom. In this case the fluctuating gauge fields are assumed electromagnetic in origin.

The fully relativistic case, examined in section 2, may admit a less conventional physical interpretation, and correspond to the case considered in [14]. There, the abelian gauge interactions represent magnetic interactions in a spin-charge separation framework for high-temperature superconductors, in which one admits intrasublattice hopping in the underlying doped antiferromagnetic model. In contrast to that work, however, which used non-relativistic fermions, here the fermions (holons) are relativistic, since one considers the spin-charge separation near the nodes. One also assumes a four-dimensional nature for such fermions, as a result of interplanar hopping. However, the above interpretation of the relativistic case is still not fully understood in the sense of not having been derived by an appropriate microscopic model at a satisfactory level of mathematical rigor.

At any rate, the generic results obtained from the field-theoretic analysis of the present article may be useful if one wishes to compare them with various scenaria that may be in operation in realistic condensed-matter situations, namely detailed phenomenological models derived from microscopic condensed-matter systems with relevance to doped antiferromagnets (and thus high-temperature superconductivity). In such cases it would be also useful to examine models where one has non-Abelian $(S U(2)$ type) fourdimensional fluctuating gauge fields, coupled to fermions, in the presence of external magnetic fields. This would be more relevant to particle-hole symmetric spin-charge separating doped models, such as those considered in [四]. We hope to come to a detailed discussion of such issues in a forthcoming publication. 


\section{Acknowledgements}

The work of J.A., K.F. and G.K. is partially supported by the TMR project FMRX-CT97-0122. That of N.E.M. is partially supported by the Leverhulme Trust (U.K.). K.F. wishes to thank Sarben Sarkar and the Physics Department of King's College London for the hospitality during the initial stages of this work.

\section{A Anisotropic Schwinger-Dyson equation}

In this Appendix we discuss the derivation of the Schwinger-Dyson equation for the fermion propagator in the anisotropic case. To this end, we shall follow the derivation given in [15] for the isotropic case. Let us first recall some definitions. Starting from the Lagrangian

$$
\mathcal{L}=-\frac{1}{4} \mathcal{F}_{\mu \nu} \mathcal{F}^{\mu \nu}+\bar{\Psi}\left[i \not \partial-g \mathcal{A}-x\left(i \partial_{3}-g \mathcal{A}_{3}\right) \gamma^{3}-m\right] \Psi,
$$

we define the connected graphs generator functional $W$ by

$$
\exp W\left[\bar{\eta}, \eta, j_{\mu}\right]=\int \mathcal{D}\left[\mathcal{A}_{\mu}, \bar{\Psi}, \Psi\right] \exp \left\{i \int_{x} \mathcal{L}+i \int_{x}\left(j^{\mu} \mathcal{A}_{\mu}+\bar{\eta} \Psi+\bar{\Psi} \eta\right)\right\}
$$

$W$ has the following functional derivatives (we do not write the space-time indices)

$$
\begin{aligned}
\frac{\delta W}{\delta j^{\mu}} & =\frac{1}{Z}\left\langle i \mathcal{A}_{\mu}\right\rangle=i A_{\mu} \\
\frac{\delta W}{\delta \bar{\eta}} & =\frac{1}{Z}\langle i \Psi\rangle=i \psi \\
W \frac{\delta}{\delta \eta} & =\frac{1}{Z}\langle i \bar{\Psi}\rangle=i \bar{\psi} \\
\frac{\delta}{\delta \bar{\eta}} W \frac{\delta \delta}{\delta \eta} & =-\bar{\psi} \psi+\frac{1}{Z}\langle\bar{\Psi} \Psi\rangle,
\end{aligned}
$$

where the expectation value $\langle\mathcal{O}\rangle$ of an operator $\mathcal{O}$ is 


$$
\langle\mathcal{O}\rangle=\int \mathcal{D}[\mathcal{A}, \bar{\Psi}, \Psi] \mathcal{O} \exp \left\{i \int_{x} \mathcal{L}+i \int_{x}(j \mathcal{A}+\bar{\eta} \Psi+\bar{\Psi} \eta)\right\},
$$

and we define

$$
(\bar{\eta} \eta) \frac{\overleftarrow{\delta}}{\delta \eta}=-\frac{\delta}{\delta \eta}(\bar{\eta} \eta)=\bar{\eta} .
$$

Inverting the relations between $\left(j_{\mu}, \bar{\eta}, \eta\right)$ and $\left(A_{\mu}, \bar{\psi}, \psi\right)$, we define the effective action $\Gamma\left[A_{\mu}, \bar{\psi}, \psi\right]$ as the Legendre transform of $W\left[j_{\mu}, \bar{\eta}, \eta\right]$ by

$$
W=i \Gamma+i \int_{x}\left(j^{\mu} A_{\mu}+\bar{\eta} \psi+\bar{\psi} \eta\right) .
$$

From this definition we extract the following functional derivatives:

$$
\begin{aligned}
\frac{\delta \Gamma}{\delta A_{\mu}} & =-j^{\mu} \\
\frac{\delta \Gamma}{\delta \bar{\psi}} & =-\eta \\
\Gamma \frac{\overleftarrow{\delta}}{\delta \psi} & =-\bar{\eta} \\
\frac{\delta}{\delta \bar{\psi}} \Gamma \frac{\grave{\delta}}{\delta \psi} & =-\frac{\delta \bar{\eta}}{\delta \bar{\psi}}=-i\left(\frac{\delta}{\delta \bar{\eta}} W \frac{\overleftarrow{\delta}}{\delta \eta}\right)^{-1} .
\end{aligned}
$$

The starting point to derive the Schwinger-Dyson equation is to assume that the integral of a derivative vanishes, such that we can write

$$
\int \mathcal{D}\left[\mathcal{A}_{\mu}, \bar{\Psi}, \Psi\right] \frac{\delta}{\delta \bar{\Psi}(z)} \exp \left\{i \int \mathcal{L}+i \int j^{\mu} \mathcal{A}_{\mu}+\bar{\Psi} \eta+\bar{\eta} \Psi\right\}=0,
$$

which leads to, after a functional derivative with respect to $\eta$ and setting $j^{\mu}=\eta=\bar{\eta}=0$,

$$
\begin{aligned}
& \delta\left(z_{1}-z_{2}\right)=\left.\left(i \partial_{z_{1}}-m-i x \partial_{3} \gamma^{3}\right) \frac{\delta}{\delta \bar{\eta}\left(z_{1}\right)} W \frac{\delta}{\delta \eta\left(z_{2}\right)}\right|_{j^{\mu}=\eta=\bar{\eta}=0} \\
& +\left.i g\left(\frac{\delta}{i \delta j^{\mu}\left(z_{1}\right)} \gamma^{\mu}-x \frac{\delta}{i \delta j^{3}\left(z_{1}\right)} \gamma^{3}\right) \frac{\delta}{\delta \bar{\eta}\left(z_{1}\right)} W \frac{\delta}{\delta \eta\left(z_{2}\right)}\right|_{j^{\mu}=\eta=\bar{\eta}=0} .
\end{aligned}
$$


We now have to turn this last equation into an equation for the effective action if we wish to obtain a relation between the proper functions. For this, we will use the last equation of Eq.(47) and we remark that

$$
\begin{aligned}
\frac{\delta \Gamma}{\delta j_{\mu}(z)}=\int d y & \left\{\frac{\delta \Gamma}{\delta A_{\nu}(y)} \frac{\delta A_{\nu}(y)}{\delta j_{\mu}(z)}+\frac{\delta \bar{\psi}(y)}{\delta j_{\mu}(z)} \frac{\delta \Gamma}{\delta \bar{\psi}(y)}+\frac{\Gamma \nless \delta}{\delta \psi(y)} \frac{\delta \psi(y)}{\delta j_{\mu}(z)}\right\} \\
=\int d y & \frac{\delta \Gamma}{\delta A_{\nu}(y)}\left(\frac{\delta^{2} \Gamma}{\delta A_{\mu}(z) \delta A_{\nu}(y)}\right)^{-1} \\
& +\left(\frac{\delta^{2} \Gamma}{\delta A_{\mu}(z) \delta \bar{\psi}(y)}\right)^{-1} \frac{\delta \Gamma}{\delta \bar{\psi}(y)} \\
& \left.+\frac{\Gamma \dot{\delta}}{\delta \psi(y)}\left(\frac{\delta \Gamma \dot{\delta}}{\delta A_{\mu}(z) \delta \psi(y)}\right)^{-1}\right\},
\end{aligned}
$$

such that we have for vanishing sources

$$
\begin{aligned}
& \left.\frac{\delta^{2} \Gamma \text { \& }}{\delta \bar{\psi}\left(z_{1}\right) \delta j_{\mu}\left(z_{2}\right) \delta \psi\left(z_{3}\right)}\right|_{\psi=\bar{\psi}=A_{\mu}=0} \\
& =\int d y \frac{\delta^{2} \Gamma \text { \& }}{\delta \bar{\psi}\left(z_{1}\right) \delta A_{\nu}(y) \delta \psi\left(z_{3}\right)}\left(\frac{\delta^{2} \Gamma}{\delta A_{\mu}\left(z_{2}\right) \delta A_{\nu}(y)}\right)^{-1} .
\end{aligned}
$$

The proper functions (respectively fermion propagator, photon propagator and vertex) are defined by

$$
\begin{aligned}
G^{-1}\left(z_{1}, z_{2}\right) & =-\left.i \frac{\delta}{\delta \bar{\psi}\left(z_{1}\right)} \Gamma \frac{\dot{\delta}}{\delta \psi\left(z_{2}\right)}\right|_{\bar{\psi}=\psi=A_{\mu}=0} \\
D_{\mu \nu}^{-1}\left(z_{1}, z_{2}\right) & =-\left.i \frac{\delta^{2} \Gamma}{\delta A^{\mu}\left(z_{1}\right) \delta A^{\nu}\left(z_{2}\right)}\right|_{\bar{\psi}=\psi=A_{\mu}=0} \\
\Lambda^{\mu}\left(z_{1} ; z_{2}, z_{3}\right) & =-\left.\frac{1}{g} \frac{\delta^{2} \Gamma \quad \delta}{\delta A_{\mu}\left(z_{1}\right) \delta \bar{\psi}\left(z_{2}\right) \delta \psi\left(z_{3}\right)}\right|_{\bar{\psi}=\psi=A_{\mu}=0}
\end{aligned}
$$

such that we finally obtain from Eq. (49) after multiplying by $S$ (we do not write the space-time indices)

$$
G=S-4 \pi \alpha \int S \gamma^{\mu} G \Lambda^{\nu} G D_{\mu \nu}+x 4 \pi \alpha \int S \gamma^{3} G \Lambda^{\nu} G D_{3 \nu}
$$

where $g^{2}=4 \pi \alpha$. 


\section{References}

[1] V.P.Gusynin, V.A.Miransky, I.A.Shovkovy, Phys.Rev. D52, 4747 (1995).

[2] D.S.Lee, C.N.Leung, Y.J.Ng, Phys.Rev. D55, 6504 (1997).

[3] For concise reviews see: V. P. Gusynin, Ukr. J. Phys. 45, 603 (2000) [hep-th/0001070], and references therein; Y. J. Ng, hep-th/9803074, and references therein. C. N. Leung, hep-th/9806208, and references therein.

[4] K. Farakos and N.E. Mavromatos, Int. J. Mod. Phys. B12, 809 (1998).

[5] K. Farakos, G. Koutsoumbas and N. E. Mavromatos, Phys. Lett. B431, 147 (1998) hep-lat/9802037; Int. J. Mod. Phys. B12, 2475 (1998) cond-mat/9805402; K. Farakos, G. Koutsoumbas, N. E. Mavromatos and A. Momen, Phys. Rev. D61, 045005 (2000) hep-ph/9905272.

[6] G. W. Semenoff, I. A. Shovkovy and L. C. Wijewardhana, Mod. Phys. Lett. A13, 1143 (1998) hep-ph/9803371; W. V. Liu, Nucl. Phys. B556, 563 (1999) cond-mat/9808134. E. J. Ferrer, V. P. Gusynin and V. de la Incera, hep-ph/0101308; V. C. Zhukovsky, K. G. Klimenko, V. V. Khudyakov and D. Ebert, JETP Lett. 73, 121 (2001) [Pisma Zh. Eksp. Teor. Fiz. 73, 137 (2001)] hep-th/0012256.

[7] V.P.Gusynin, I.A.Shovkovy, Phys. Rev. D56, 5251 (1997); D.S.Lee, C.N.Leung, Y.J.Ng, Phys. Rev. D57, 5224 (1998).

[8] K. Krishana et al., Science 277, 83 (1997).

[9] V.P. Gusynin, V.A. Miransky, I.A. Shovkovy, Nucl. Phys. B563, 361 (1999).

[10] P. W. Anderson, Science 235, 1196 (1987); G. Baskaran, Z. Zou and P.W. Anderson, Solid. State Comm. 63, 973 (1987); G. Baskaran and P.W. Anderson, Phys. Rev. B37, 580 (1988); R.B. Laughlin, Science 242, 525 (1988).

[11] K. Farakos and N.E. Mavromatos, Phys. Rev. B57, 3017 (1998).

[12] E. V. Gorbar, V. P. Gusynin and V. A. Miransky, hep-ph/0105059. 
[13] J. Alexandre, K. Farakos, G. Koutsoumbas, Phys.Rev. D63, 065015 (2001).

[14] L. Ioffe and P. Wiegmann, Phys. Rev. Lett. 65, 653 (1990).

[15] C. Itzyckson, J.-B. Zuber, Quantum Field Theory, Mc Graw-Hill (1980), section 10.1.1. 